\title{
The comparative energetics of the turtles and crocodiles
}

\author{
Nina Marn ${ }^{1}$ and Sebastiaan Kooijman ${ }^{2}$ \\ ${ }^{1}$ Rudjer Boskovic \\ ${ }^{2}$ Vrije Universiteit Amsterdam
}

November 12, 2021

\begin{abstract}
The Add-my-Pet (AmP) collection of data on energetics and Dynamic Energy Budget (DEB) parameters currently contains 92 species of turtles and 23 species of crocodiles. We discuss patterns of eco-physiological traits of turtles and crocodiles, as functions of parameter values, and compare them with other taxa. Turtles and crocodiles accurately match the general rule that the life-time cumulated neonate mass production equals ultimate weight. The weight at birth for reptiles scales with ultimate weight to the power 0.6. The scaling exponent is between that of amphibians and birds, while that for mammals is close to 1 . We explain why this points to limitations imposed by embryonic respiration, the role of water stress and the accumulation of nitrogen waste during the embryo stage. Weight at puberty is proportional to ultimate weight, and is the largest for crocodiles, followed by that of turtles. These facts explain why the precociality coefficient - approximated by the ratio of weight at birth and weight at puberty at abundant food - decreases with ultimate weight. It is the smallest for crocodile,s because of their large size, while that lizards and snakes are much larger than for turtles. The maximum reserve capacity in both turtles and crocodiles clearly decreases with the precociality coefficient. This relationship has not be found that clearly in other taxa, not even in other reptiles. Crocodiles have a relatively large assimilation rate and, as consequence, a large reserve capacity. Sea-turtles have a small weight and age at birth, which we link to reducing risks on the beach.
\end{abstract}

\section{Hosted file}

MarnKooy2021.pdf available at https://authorea.com/users/445757/articles/545169-thecomparative-energetics-of-the-turtles-and-crocodiles 